\title{
Exponential Growth for the Wave Equation with Compact Time-Periodic Positive Potential
}

\author{
FERRUCCIO COLOMBINI \\ Università di Pisa \\ VESSELIN PETKOV \\ Institut de Mathématiques de Bordeaux \\ AND \\ JEFFREY RAUCH \\ University of Michigan
}

\begin{abstract}
We prove the existence of smooth positive potentials $V(t, x)$, periodic in time and with compact support in $x$, for which the Cauchy problem for the wave equation $u_{t t}-\Delta_{x} u+V(t, x) u=0$ has solutions with exponentially growing global and local energy. Moreover, we show that there are resonances, $z \in \mathbb{C}$, $|z|>1$, associated to $V(t, x)$. (c) 2008 Wiley Periodicals, Inc.
\end{abstract}

\section{Introduction}

Consider the Cauchy problem for the wave equation with potential

$$
\left\{\begin{array}{l}
\partial_{t}^{2} u-\Delta_{x} u+V(t, x) u=0, \quad(t, x) \in \mathbb{R}^{1+3}, \\
\left.u\right|_{t=s}=f_{1}(x),\left.\quad u_{t}\right|_{t=s}=f_{2}(x) .
\end{array}\right.
$$

The potential, $V \in C^{\infty}\left(\mathbb{R}^{1+3} ; \mathbb{R}\right)$, is time periodic and compactly supported, that is,

$$
\begin{aligned}
& \exists \rho, T>0, \forall(t, x) \in \mathbb{R}^{1+3}, \\
& V(t+T, x)=V(t, x), \quad \operatorname{supp} V \subset\{|x| \leq \rho\} .
\end{aligned}
$$

Denote by $D$ the closure of $C_{0}^{\infty}\left(\mathbb{R}^{3}\right)$ with respect to the norm

$$
\|\varphi\|_{D}:=\left(\int_{\mathbb{R}^{3}}|\nabla \varphi(x)|^{2} d x\right)^{1 / 2} .
$$

For initial data $f=\left(f_{1}, f_{2}\right)$ in the Hilbert space $H:=D \times L^{2}\left(\mathbb{R}^{3}\right)$ there is a unique solution $u \in C(\mathbb{R} ; D) \cap C^{1}\left(\mathbb{R} ; L^{2}\left(\mathbb{R}^{3}\right)\right)$ of (1.1). Define the propagator $U(t, s) f:=\left(u, u_{t}\right)(t, x)$ and denote by $U_{0}(t, s)$ the propagator in the case $V=0$. We denote by $(\cdot, \cdot)_{H}$ the scalar product in $H$. When $V=0$ the coefficients are independent of time, so the propagator $U_{0}(t, s)$ depends only on $t-s$. 
For

one has the differential energy law

$$
e:=\frac{1}{2}\left(\left|u_{t}\right|^{2}+\left|\nabla_{x} u\right|^{2}\right)
$$

$$
\partial_{t} e-\operatorname{div}_{x}\left(\operatorname{Re}\left(\bar{u}_{t} \nabla_{x} u\right)\right)=2 \operatorname{Re}\left(\bar{u}_{t}\left(u_{t t}-\Delta u\right)\right) .
$$

Integrating over $\mathbb{R}_{x}^{3}$ when $V=0$ shows that $U_{0}$ is unitary.

Since $V$ is compactly supported there is a constant $\alpha$ depending only on $V$ so that

$$
\|V u\|_{H^{1}\left(\mathbb{R}^{3}\right)} \leq \alpha\left\|\nabla_{x} u\right\|_{L^{2}\left(\mathbb{R}^{3}\right)} .
$$

Estimating

$$
\left|\int \bar{u}_{t} V u d x\right| \leq\left\|u_{t}\right\|_{L^{2}\left(\mathbb{R}^{3}\right)}\|V u\|_{L^{2}\left(\mathbb{R}^{3}\right)} \leq \alpha\left\|u_{t}\right\|_{L^{2}\left(\mathbb{R}^{3}\right)}\left\|\nabla_{x} u\right\|_{L^{2}\left(\mathbb{R}^{3}\right)}
$$

shows that for solutions of (1.1),

$$
\partial_{t} \int e(t, x) d x \leq 2 \alpha \int e(t, x) d x,
$$

whence

$$
\|U(t, s) f\|_{H} \leq e^{\alpha|t-s|}\|f\|_{H} .
$$

The perturbation $U(t, s) f-U_{0}(t, s) f$ is equal to $\left(w(t), w_{t}(t)\right)$, where $w$ is the unique solution of the Cauchy problem

$$
w_{t t}-\Delta w=-V u,\left.\quad w\right|_{t=s}=\left.w_{t}\right|_{t=s}=0 .
$$

Estimate (1.3) shows that $V u \in C\left(\mathbb{R} ; H^{1}\left(\mathbb{R}^{3}\right)\right)$ and $V u$ is supported in $|x| \leq \rho$. It follows that

$w \in C\left(\mathbb{R} ; H^{2}\left(\mathbb{R}^{3}\right)\right) \cap C^{1}\left(\mathbb{R} ; H^{1}\left(\mathbb{R}^{3}\right)\right), \quad \operatorname{supp} w(t) \subset\{|x| \leq \rho+|t-s|\}$,

$\exists C, \quad \forall|t-s| \leq T, \quad\left\|\left(w(t), w_{t}(t)\right)\right\|_{H^{2}\left(\mathbb{R}^{3}\right) \times H^{1}\left(\mathbb{R}^{3}\right)} \leq C\left\|\left(u(s), u_{t}(s)\right)\right\|_{H}$.

In particular, $U(t, s)$ is a compact perturbation of the unitary operator $U_{0}(t, s)$. Therefore, the spectrum of the Floquet or monodromy operator $U(T, 0)$ consists of the essential spectrum equal to the unit circle and at most a countable set of eigenvalues of finite algebraic multiplicity. In the present context, it is known that there are at most a finite number of eigenvalues with modulus greater than 1 [12].

These results correspond to the intuitive idea that typical waves radiate to infinity, escaping the region where the potential can act. For large time such solutions behave like solutions of the free wave equation. The pure point spectrum off the unit circle yields exceptional solutions that do not radiate to infinity.

If there is a point $\underline{z} \in \operatorname{spec} U(T, 0)$ with $|\underline{z}|>1$, then choosing $f$ as an eigenfunction one has

$$
U(n T, 0) f=\underline{z}^{n} f, \quad n \in \mathbb{Z} .
$$

Taking $n \rightarrow \infty$ shows that (1.4) cannot hold with $\alpha=0$, and there are solutions that grow exponentially in time. 
It is easy to construct examples of growing solutions when the potential $V$ is permitted to take negative values [7, 10]. For example, if $C_{0}^{\infty}\left(\mathbb{R}^{3}\right) \ni V=V(x) \leq$ 0 and is not identically equal to 0 , then for $g>0$ sufficiently large, the Schrödinger operator $-\Delta+g V$ has a strictly negative eigenvalue

$$
(-\Delta+g V) \psi=-\lambda^{2} \psi, \quad \psi \in \mathcal{S}\left(\mathbb{R}^{3}\right), \quad \lambda>0 .
$$

Then $u(t, x):=e^{\lambda t} \psi(x)$ is an exponentially growing solution. The propagator $U(T, 0)$ for the potential $g V(x)$ satisfies

$$
U(T, 0)(\psi, \lambda \psi)=e^{\lambda T}(\psi, \lambda \psi), \quad(\psi, \lambda \psi) \in H .
$$

Consider the perturbed potential $g V(x)+\epsilon W(t, x)$ with $W$ smooth, $T$ periodic, and compactly supported. The new evolution operator $U^{\epsilon}(T, 0)$ satisfies $\left\|U^{\epsilon}(T, 0)-U(T, 0)\right\| \leq C \epsilon$. So for $\epsilon$ small it has a pure point spectrum near $e^{\lambda T}$. When $V \geq 0$ the situation is radically different. For the time-independent case, $V=V(x)$, the energy

$$
\int\left[e(t, x)+V(x) \frac{|u(t, x)|^{2}}{2}\right] d x
$$

is conserved and there is no growth.

When Cooper and Strauss extended the Lax-Phillips theory to time-periodic scatterers $[8,9]$ more than 30 years ago, they conjectured that there exist periodic potentials $V \geq 0$ with compact support so that $\operatorname{spec} U(T, 0) \cap\{|z|>1\} \neq \varnothing$. Numerical computations $[15,17,18]$ supported this conjecture. The conjecture was further supported in Cooper [6], where certain perturbations were proved to lead to slow decay. Cooper conjectured that larger perturbations could lead to growth. Our main result proves the conjecture of Cooper and Strauss.

THEOREM 1.1 There exists a nonnegative, smooth, T-periodic, compactly supported potential $V(t, x)$ for which the Floquet operator $U(T, 0)$ has an eigenvalue of modulus greater than 1.

There are other constructions of growing solutions preceding ours, each failing for the case of smooth, compact, positive periodic potentials. If one eschews compact support, then one can choose $V=V(t)$ independently of $x$. A Fourier transform in $x$ transforms the problem to

$$
\hat{u}_{t t}(t, \xi)+|\xi|^{2} \hat{u}(t, \xi)+V(t) \hat{u}(t, \xi)=0 .
$$

One can then choose $V \geq 0$ smooth and periodic so that there are $\xi$ such that the Hill's operator $d^{2} / d t^{2}+\left(V(t)+|\xi|^{2}\right)$ has exponentially growing solutions. Such growth when the frozen operators have strictly positive conserved energies is called parametric resonance. Choosing data whose Fourier transform is supported in intervals of $\xi$ where such resonance occurs yields growing solutions.

For the wave equation with potential, the bicharacteristics are exactly the same as those of the free wave equation. In particular, they lie over rays in $(t, x)$ that 
travel along straight lines at speed 1 . These rays escape the region where the potential acts and the problem is nontrapping. There are examples of exponentially growing solutions with compactly supported periodic perturbations that trap rays.

The Dirichlet problem for periodically moving obstacles having trapped rays is examined in [5, 14]. In particular, in [14] it was proved that if we have at least one bicharacteristic with suitable amplifying properties, then there exist solutions with exponentially growing local energy. The existence of a trapped bicharacteristic is exploited in [4] for the Cauchy problem for the equation

$$
\partial_{t}^{2} u-\operatorname{div}_{x}(a(t, x) \operatorname{grad} u)=0,
$$

where $a(t, x)$ is periodic in time, $0<c \leq a(t, x) \leq C$, and $a(t, x)=1$ for $|x| \geq$ $\rho$. The growth associated to these trapped rays is connected with the presence of a continuous spectrum of the monodromy operator $U(T, 0)$ outside the unit disc. In that regard, recall the conjecture (see [13]) that for trapping periodic perturbations, the cutoff resolvent $\chi(x)(U(T, 0)-z I)^{-1} \chi(x)$ with $\chi \in C_{0}^{\infty}\left(\mathbb{R}^{3}\right)$ equal to 1 for $|x| \leq \rho+T$ does not have a meromorphic continuation from $\{z \in \mathbb{C}:|z| \geq A \gg$ $1\}$ to $\{|z|>1\}$.

Our strategy is to confine and pump. The pump is based on the equation with potential $q(t)$ in the ball $\{|x| \leq L\}$ and Dirichlet boundary conditions on the boundary, $\{|x|=L\}$. Expanding in eigenfunctions of the Dirichlet Laplacian yields Hill's equations

$$
a_{n}^{\prime \prime}(t)+q(t) a_{n}(t)+\lambda_{n} a_{n}(t)=0
$$

for the Fourier coefficients $a_{n}(t)$. One chooses $q(t)$ so that one of these Hill's equations has exponentially growing solutions. Choose a sequence of cutoff functions $\chi^{\delta}(x) \in C_{0}^{\infty}(|x|<L), 0 \leq \chi \leq 1$, with $\chi=1$ except in a layer of width $\delta$ of the boundary. Replacing the potential $q(t)$ by $q(t) \chi^{\delta}(x)$ changes the evolution operator little in norm, so the operator $K^{\delta}(T)$ taking Cauchy data at $t=0$ to data at $t=T$ has an eigenvalue $z_{1}$ of modulus greater than 1 . That is the pump.

In the next step, replace the Dirichlet condition by a potential $b^{\epsilon}(x)$ where $b^{\epsilon}(x) \in C_{0}^{\infty}\left(\mathbb{R}^{3}\right)$ is a barrier supported in $\{L \leq|x| \leq L+1\}$ and is equal to $\frac{1}{\epsilon}$ for $\{L+\epsilon \leq|x| \leq L+1-\epsilon\}$. Let $V^{\epsilon}(t, x):=b^{\epsilon}(x)+q(t) \chi^{\delta}(x)$ with $\epsilon>0$. When $\epsilon$ is sufficiently small, initial waves supported in $|x| \leq L$ are nearly confined to that ball. There is leakage through the barrier. We prove that the growth from the pump beats the loss by showing that there is an eigenvalue of modulus greater than 1 .

Our construction is inspired by the analysis of Beale [2] for the Helmholtz resonator. For $z_{1}$ and $\varphi$, the eigenvalue and eigenfunction of $K^{\delta}(T)$, the main difficulty is to prove that the resolvents $\left(K^{\delta}(T)-z I\right)^{-1} \varphi$ and $\left(U^{\epsilon}(T, 0)-z I\right)^{-1} \varphi$ for $z$ belonging to a small circle $\gamma$ centered at $z_{1}$ are close. Since $K^{\delta}(T)$ has an eigenvalue $z_{1},\left|z_{1}\right|>1$, the Cauchy contour integral of its resolvent over $\gamma$ is 
nonzero. It follows that the integral of the resolvent of $U^{\epsilon}(0, T)$ over $\gamma$ is nonzero, proving the existence of an eigenvalue $z_{\epsilon},\left|z_{\epsilon}\right|>1$, of $U^{\epsilon}(0, T)$. For the problem of Beale with time-independent coefficients, it sufficed to compare the resolvents of the generators. For that, the analysis leaned heavily on elliptic regularity. The heart of our proof is the convergence of

$$
\left.\left(\varphi,\left(U^{\epsilon}(T, 0)\right)-z I\right)^{-1} \varphi\right)_{H} \rightarrow\left(\varphi,\left(K^{\delta}(T)-z I\right)^{-1} \varphi\right)_{H}
$$

whose proof is more delicate.

For problem (1.1) there is a notion of resonance, and a corollary of Theorem 1.1 proves the existence of resonances. The existence of such resonances for positive, compact, periodic potentials is conjectured in the same articles cited above. Recall three equivalent definitions of resonances (see $[3,12]$ for details concerning resonances). The first definition concerns smooth solutions $u \in C^{\infty}\left(\mathbb{R} ; \mathcal{D}^{\prime}\left(\mathbb{R}^{3}\right)\right)$ of (1.1) that, though not necessarily small for $|x| \rightarrow \infty$, are outgoing.

Outgoing solutions are defined as follows: For $\Phi \in C_{0}^{\infty}\left(\mathbb{R}^{3}\right) \times C_{0}^{\infty}\left(\mathbb{R}^{3}\right)$ denote $U_{0}(t, 0) \Phi=\left(w_{\Phi}(t), \partial_{t} w_{\Phi}(t)\right)$. A solution $u$ is outgoing when for all $\Phi$,

$$
\lim _{t \rightarrow-\infty}\left(\left\langle u(t), w_{\Phi}(t)\right\rangle+\left\langle u_{t}(t), \partial_{t} w_{\Phi}(t)\right\rangle\right)=0
$$

where $\langle\cdot, \cdot\rangle$ means the action in the sense of distributions. A point $z=e^{i \sigma T}$ is a resonance if there exists an outgoing solution $u(t, x)$ of (1.1) with data $0 \neq f=$ $\left(f_{1}, f_{2}\right) \in H$ such that $e^{-i \sigma t} u(t, x)$ is periodic in $t$ with period $T$. In this case $\sigma \in \mathbb{C}$ is called a scattering frequency.

The second definition concerns cutoff resolvents. Suppose that $\chi \in C_{0}^{\infty}\left(\mathbb{R}^{3}\right)$ is identically equal to 1 on a neighborhood of $\{x: \operatorname{dist}(x, \operatorname{supp} V) \leq T\}$. Then the cutoff resolvent $\chi(U(T, 0)-z)^{-1} \chi$ has a meromorphic extension from $|z| \gg 1$ to $z \neq 0$ and the poles are the resonances.

Finally, the resonances $z \neq 0$ are the eigenvalues of the reduced Floquet operator

$$
Z^{b}(T, 0):=P_{+}^{b} U(T, 0) P_{-}^{b},
$$

where $P_{ \pm}^{b}$ are the orthogonal projections on the orthogonal complements of LaxPhillips [10] spaces,

$$
D_{ \pm}^{b}:=\left\{f \in H: U_{0}(t) f=0 \text { when }|x| \leq b \pm t, \pm t \geq 0\right\}, \quad b>\rho .
$$

The spectrum of $Z^{b}(T, 0)$ is independent of $b>\rho$, and in $\mathbb{C} \backslash\{0\}$ it consists of a discrete set of eigenvalues with finite multiplicity that can only accumulate at 0 .

For $T$-periodic, compactly supported, smooth $V$, it is known that the following are equivalent [12, theorem 5.5.3]: 
(1) One has local energy decay in the sense that for any $\chi \in C_{0}^{\infty}\left(\mathbb{R}^{3}\right)$ the operator $\chi U(t, 0) \chi$ tends strongly to 0 as $t \rightarrow \infty$.

(2) One has exponential decay of local energy in the sense that for any $\chi \in$ $C_{0}^{\infty}\left(\mathbb{R}^{3}\right)$ there is a constant $C=C(\chi)>0$ so that for $t \geq 0$,

$$
\|\chi U(t, 0) \chi\|_{\mathcal{L}(H)} \leq C e^{-C t} .
$$

(3) The operator $Z^{b}(T, 0)$ has no eigenvalues $z$ with $|z| \geq 1$.

For related results concerning the local energy decay, see $[1,16]$.

The dichotomy given by the presence or absence of eigenvalues of $Z(T, 0)$ of modulus greater than or equal to 1 determines whether the local energy decays. There are some sufficient conditions guaranteeing the absence of such a point spectrum that do not pretend to be sharp [12]. Theorem 5.4.1 in [12] proves that for $|\lambda|>1$, the operator $P_{+}^{a}, a>\rho$, is an isomorphism from the generalized eigenspace $\mathcal{G}_{\lambda}$ of $U(T, 0)$ corresponding to $\lambda$ to the generalized eigenspace $\mathcal{F}_{\lambda}$ of $Z(T, 0)$ corresponding to $\lambda$. Thus $Z(T, 0)$ has no eigenvalue $\lambda,|\lambda|>1$, if and only if $U(T, 0)$ has no such eigenvalue and we obtain the following:

COROLLARY 1.2 There exists a nonnegative, smooth, T-periodic, compactly supported potential $V(t, x)$ for which problem (1.1) has a resonance with modulus greater than 1.

\section{Remark 1.3.}

(i) Our analysis of the confine and pump mechanism works for general domains and dimensions. For ease of reading, we present the case of a ball in $\mathbb{R}^{3}$.

(ii) In Section 5 we give a proof, independent of [12], that there are resonances near the eigenvalues of the pump.

The paper is organized as follows: Section 2 is devoted to studying the pump. Section 3 proves the weak convergence on space-time of the solutions of the equation with potentials $V^{\epsilon}$ to those of the uncoupled Dirichlet problems for suitably restricted, weakly convergent Cauchy data. In Section 4 we prove weak convergence of the resolvent $\left(U^{\epsilon}(T, 0)-z I\right)^{-1} \varphi$ to the corresponding resolvent of the decoupled Dirichlet problems when $\varphi$ vanishes for $L \leq|x| \leq L+1$ and $z$ does not meet the spectra of $U^{\epsilon}(T, 0)$. The key step is a bound on the resolvents independent of $\epsilon$. The main results are derived from this in Section 5.

\section{The Pump}

Define $B_{L}:=\left\{x \in \mathbb{R}^{3}:|x| \leq L\right\}$. The starting point of the construction is the mixed initial boundary value problem in $\mathbb{R}_{t} \times B_{L}$,

$$
\left\{\begin{array}{l}
u_{t t}-\Delta_{x} u+q(t) u=0, \quad(t, x) \in \mathbb{R} \times B_{L}, \\
\left.u(t, x)\right|_{|x|=L}=0
\end{array}\right.
$$


Choose a $T$-periodic smooth potential $q(t) \geq 0$ such that the equation

$$
a^{\prime \prime}(t)+q(t) a(t)+\lambda a(t)=0
$$

has an interval of instability $I=] \alpha, \beta\left[\subset \mathbb{R}^{+}\right.$(see, for instance, [11]). Choose $L>0$ and $k \in \mathbb{N}$ so that $\lambda=k^{2} \pi^{2} / L^{2} \in I$. Then there is a $\mu_{1}$ with $\left|\mu_{1}\right|>1$ and a solution $a(t)$ of (2.2) so that

$$
\left(a(T), a^{\prime}(T)\right)=\mu_{1}\left(a(0), a^{\prime}(0)\right) .
$$

Fix $k$ and $L$. Then

$$
u(t, x):= \begin{cases}|x|^{-1} a(t) \sin (k \pi|x| / L) & \text { if } x \neq 0, \\ k \pi a(t) / L & \text { if } x=0,\end{cases}
$$

is a solution of (2.1) that is exponentially growing as $t \rightarrow+\infty$.

Denote by $K(T)$ the operator taking Cauchy data $\left(u_{0}, u_{1}\right) \in H_{0}^{1}\left(B_{L}\right) \times L^{2}\left(B_{L}\right)$ at time $t=0$ to Cauchy data $\left(u(T), u_{t}(T)\right)$ at time $T$. Denote by $K_{0}(T)$ the analogous operator for the problem with $q=0$. Then $K_{0}(T)$ is unitary in the norm

$$
\left\|\left(u(t), u_{t}(t)\right)\right\|^{2}:=\frac{1}{2} \int_{B_{L}}\left(\left|\nabla_{x} u(t, x)\right|^{2}+\left|u_{t}(t, x)\right|^{2}\right) d x .
$$

The operator $K(T)$ is a compact perturbation of $K_{0}(T)$ and, by construction, $K(T)$ has an isolated eigenvalue $\mu_{1}$ with $\left|\mu_{1}\right|>1$.

For each $0<\delta<\frac{L}{10}$, choose $\chi^{\delta}(x) \in C_{0}^{\infty}\left(B_{L}\right)$ with

$$
0 \leq \chi^{\delta}(x) \leq 1,\left.\quad \chi^{\delta}\right|_{B_{L-\delta}}=1 .
$$

Denote by $K^{\delta}(T) \in \operatorname{Hom}\left(H_{0}^{1}\left(B_{L}\right) \times L^{2}\left(B_{L}\right)\right)$ the evolution operator associated to the differential operator $\partial_{t}^{2}-\Delta_{x}+q(t) \chi^{\delta}(x)$ with Dirichlet boundary conditions on $|x|=L$. The energy method shows that as $\delta \rightarrow 0$, we have

$$
\left\|K^{\delta}(T)-K(T)\right\|_{\mathrm{Hom}\left(H_{0}^{1}\left(B_{L}\right) \times L^{2}\left(B_{L}\right)\right)} \rightarrow 0 .
$$

Choose $r<\left|\mu_{1}\right|-1$ so that $\mu_{1}$ is the only eigenvalue of $K(T)$ belonging to the disc $D_{r}$ of radius $r$ and center $\mu_{1}$. The norm convergence (2.3) implies that one can choose $0<\delta \ll 1$ so that $K^{\delta}(T)$ has at least one eigenvalue $z_{1}$ inside the disc $D_{r}$ and no eigenvalue on the boundary $\partial D_{r}$. The eigenvalue $z_{1}$ may have multiplicity greater than 1 .

In the following we will assume that $0<\delta \ll 1$ is fixed so that $K^{\delta}(T)$ has an eigenvalue $z_{1} \in D_{r}$. Consider the wave equation

$$
u_{t t}-\Delta_{x} u+V^{\epsilon}(t, x) u=0, \quad V^{\epsilon}(t, x):=b^{\epsilon}(x)+q(t) \chi^{\delta}(x),
$$

with barrier potential

$0 \leq b^{\epsilon}(x) \in C_{0}^{\infty}(\{L<|x|<L+1\}), \quad b^{\epsilon}(x)=\frac{1}{\epsilon}$ on $\{L+\epsilon<|x|<L+1-\epsilon\}$. 
For Cauchy data

$$
\left(u(0, x), u_{t}(0, x)\right)=w=\left(w_{1}(x), w_{2}(x)\right),
$$

which are supported in $B_{L}$, and for $\epsilon>0$ sufficiently small, the solution at time $T$ is mostly confined to $\{|x| \leq L\}$ and is well approximated by the solution given as the extension by 0 of $K^{\bar{\delta}}(T) w$. We prove that for $0<\epsilon \ll 1$ and $t=T$ the evolution operator $U^{\epsilon}(T, 0)$ for (2.4) has an eigenvalue inside $D_{r}$.

\section{Weak Convergence}

The first step is to study the weak limits as $\epsilon \rightarrow 0$ of the differential equations on $\mathbb{R}^{1+3}$ with potentials $V^{\epsilon}$. Introduce the energy

$$
E^{\epsilon}(u, t):=\frac{1}{2} \int_{\mathbb{R}^{3}}\left(\left|\nabla_{t, x} u(t, x)\right|^{2}+b^{\epsilon}(x)|u(t, x)|^{2}\right) d x .
$$

When there is little risk of confusion, the dependence on $u$ will be suppressed. For $t>0$, the energy identity implies

$$
E^{\epsilon}(t)-E^{\epsilon}(0)=-2 \operatorname{Re} \int_{0}^{t} \int_{\mathbb{R}^{3}} q \chi^{\delta} u \bar{u}_{t} d t d x .
$$

For $\left(w_{1}, w_{2}\right) \in D \times L^{2}\left(\mathbb{R}^{3}\right)=H$ we use two norms,

$$
\left\|\left(w_{1}, w_{2}\right)\right\|_{\epsilon}^{2}=\int_{\mathbb{R}^{3}}\left(\left|w_{2}\right|^{2}+\left|\nabla_{x} w_{1}\right|^{2}+b^{\epsilon}\left|w_{1}\right|^{2}\right) d x,
$$

and

$$
\left\|\left(w_{1}, w_{2}\right)\right\|_{H}^{2}=\int_{\mathbb{R}^{3}}\left(\left|w_{2}\right|^{2}+\left|\nabla_{x} w_{1}\right|^{2}\right) d x .
$$

Denote by $D_{0}$ the elements of $D$ that vanish for $L \leq|x| \leq L+1$. On $D_{0} \times L^{2}\left(\mathbb{R}^{3}\right)$ the norms $\|\cdot\|_{H}$ and $\|\cdot\|_{\epsilon}$ are equal. A function $u \in C\left(\mathbb{R} ; D_{0}\right)$ vanishes on $|x|=L$ and $|x|=L+1$. It is in this way that Dirichlet conditions at these boundaries are expressed in the following proposition:

Proposition 3.1 Consider a sequence $\epsilon_{n} \rightarrow 0$ and weakly convergent Cauchy data in $H$

$$
w^{n}=\left(w_{1}^{n}, w_{2}^{n}\right) \rightarrow w \in H .
$$

Suppose in addition that

$$
\exists C>0, \quad \forall n, \quad\left\|w^{n}\right\|_{\epsilon_{n}} \leq C .
$$

Denote by $u^{n}$ the solution of equation (2.4) with initial data $w^{n}$. Then for any $\underline{T}>0$ there exists $u$ so that

$$
u^{n} \rightarrow u \quad \text { weak-star in } L^{\infty}([0, T] ; D)
$$


and

$$
\partial_{t} u^{n} \rightarrow \partial_{t} u \quad \text { weak-star in } L^{\infty}\left([0, \underline{T}] ; L^{2}\left(\mathbb{R}^{3}\right)\right) .
$$

Moreover, $u(t) \in C\left(\mathbb{R} ; D_{0}\right) \cap C^{1}\left(\mathbb{R} ; L^{2}\left(\mathbb{R}^{3}\right)\right)$ and $u(t)$ for $|x| \leq L$ is the unique solution of the Dirichlet problem in $\mathbb{R} \times B_{L}$

$$
u_{t t}-\Delta_{x} u+q(t) \chi^{\delta}(x) u=0, \quad\left(u(0), u_{t}(0)\right)=\left.w\right|_{B_{L}},\left.\quad u\right|_{|x|=L}=0,
$$

while $u(t)$ for $|x| \geq L+1$ is the unique solution of the Dirichlet problem in $\mathbb{R} \times\{x:|x| \geq L+1\}$

$$
u_{t t}-\Delta_{x} u=0, \quad\left(u(0), u_{t}(0)\right)=\left.w\right|_{\{|x| \geq L+1\}},\left.\quad u\right|_{|x|=L+1}=0 .
$$

Remark 3.2. The values of the initial time derivative $\left.\partial_{t} w^{n}\right|_{L \leq|x| \leq L+1}$ on $\{L \leq$ $|x| \leq L+1\}$ do not influence the limit.

Proof: Fix $\underline{T}>0$. The energy estimate shows that $u^{n}$ is bounded in $L^{\infty}([0, T] ; D)$ and $\partial_{t} u^{n}$ is bounded in $L^{\infty}\left([0, T] ; L^{2}\left(\mathbb{R}^{3}\right)\right)$. It suffices to show that any weak-star limit point vanishes for $L \leq|x| \leq L+1$ and satisfies equations (3.2) and (3.3).

Passing to a subsequence, we can suppose that $u^{n}$ converges weak-star to $v$, and $\partial_{t} u^{n}$ converges weak-star to $\partial_{t} v$ in $L^{\infty}([0, T] ; D)$ and $L^{\infty}\left([0, T] ; L^{2}\left(\mathbb{R}^{3}\right)\right)$, respectively. The energy estimate implies that $v=0$ for $L \leq|x| \leq L+1$. That is, $v$ takes values in $D_{0}$. Therefore $v_{t}=0$ for $L \leq|x| \leq L+1$.

To identify the limit in $\{|x| \geq L+1\}$ where the lower-order terms vanish, suppose that $\varphi \in C_{0}^{\infty}\left(\mathbb{R}_{t} \times \mathbb{R}_{x}^{3}\right)$ vanishes when $|x|=L+1$ and $t \geq \underline{T}$. An integration by parts shows that

$$
\begin{aligned}
\int_{0}^{\underline{T}} & \int_{|x| \geq L+1} u^{n}\left(\partial_{t}^{2}-\Delta_{x}\right) \varphi d t d x \\
= & \int_{0}^{\underline{T}} \int_{|x|=L+1} u^{n}(t, x) \varphi_{r}(t, x) d t d S_{x} \\
& +\int_{|x| \geq L+1}\left(w_{1}^{n} \varphi(0, x)-w_{2}^{n} \partial_{t} \varphi(0, x)\right) d x,
\end{aligned}
$$

where $\varphi_{r}$ denotes the radial derivative of $\varphi$.

Since $u^{n}$ is weakly convergent in $L^{2}\left([0, T] ; H_{\text {loc }}^{1}\{|x| \leq L+1\}\right)$, its trace on $|x|=L+1$ converges weakly in $L^{2}\left([0, T] ; L^{2}(\{|x|=L+1\})\right)$ to the trace of $v$. On the other hand, since the limit $v$ vanishes for $L \leq|x| \leq L+1$, the trace of $v$ vanishes, so passing to the limit yields

$$
\int_{0}^{\underline{T}} \int_{|x| \geq L+1} v\left(\partial_{t}^{2}-\Delta_{x}\right) \varphi d t d x=\int_{|x| \geq L+1}\left(w_{1} \varphi(0, x)-w_{2} \partial_{t} \varphi(0, x)\right) d x
$$


This is the weak form of equation (3.2) with the Dirichlet boundary condition on $|x|=L+1$. Uniqueness for that problem shows that $v$ is equal to the advertised $u$ on that set.

To identify the limit $v$ in $|x| \leq L$, suppose that $\psi \in C^{\infty}\left(\mathbb{R}_{t} \times B_{L}\right)$ vanishes when $|x|=L$ and for $t \geq T$. Integration by parts shows that

$$
\begin{aligned}
& \int_{0}^{\underline{T}} \int_{B_{L}} u^{n}\left(\partial_{t}^{2}-\Delta_{x}+q(t) \chi^{\delta}(x)\right) \psi d t d x \\
& =\int_{B_{L}}\left(w_{1}^{n}(x) \psi(0, x)-w_{2}^{n}(x) \psi_{t}(0, x)\right) d x \\
& \quad+\int_{0}^{\underline{T}} \int_{|x|=L} u^{n}(t, x) \psi_{r}(t, x) d t d S_{x}+\int_{0}^{\underline{T}} \int_{B_{L}} u^{n} q(t) \chi^{\delta}(x) \psi d t d x .
\end{aligned}
$$

Passing to the limit in the first line is easy. On the other hand, we have shown that the traces of $u^{n}$ on $\{x:|x|=L\}$ tend to 0 weakly in $L^{2}\left([0, T] ; L^{2}(\{|x|=\right.$ $L\}))$. Thus, passing to the limit, we find

$$
\begin{aligned}
& \int_{0}^{\frac{T}{T}} \int_{B_{L}} v\left(\partial_{t}^{2}-\Delta_{x}+q(t) \chi^{\delta}(x)\right) \psi d t d x= \\
& \quad \int_{B_{L}}\left(w_{1}(x) \psi_{x}(0, x)-w_{2}(x) \psi_{t}(0, x)\right) d x+\int_{0}^{\underline{T}} \int_{B_{L}} v q(t) \chi^{\delta}(x) \psi d t d x .
\end{aligned}
$$

This is the weak form of the initial boundary value problem (3.3) with Dirichlet boundary conditions on $\{x:|x|=L\}$. This uniquely determines the restriction of $v$ to $B_{L}$ as the solution of (3.3).

Proposition 3.1 proves weak convergence in regions of space-time. The next example shows that the sequence of solutions $u^{n}(t)$ need not converge weakly for $t$ fixed.

EXAMPLE If $w_{1}^{n}=0$ and $w_{2}^{n}=1$ on a neighborhood of the sphere $\left\{|x|=L+\frac{1}{2}\right\}$ in the center of the barrier, then finite speed of propagation implies that on a spacetime neighborhood of $\{t=0\} \times\left\{|x|=L+\frac{1}{2}\right\}, u^{n}(t, x)=y^{n}(t)$ where $y^{n}$ is the solution of the ordinary differential equation

$$
\frac{d^{2} y^{n}}{d t^{2}}+\frac{y^{n}}{\epsilon_{n}}=0, \quad y^{n}(0)=0, \quad \frac{d y^{n}}{d t}(0)=1
$$

Then, $y^{n}(t)=\sqrt{\epsilon_{n}} \sin \left(t / \sqrt{\epsilon_{n}}\right), d y^{n} / d t=\cos \left(t / \sqrt{\epsilon_{n}}\right)$. Thus for typical $\epsilon_{n} \rightarrow$ $0, d y^{n}(t) / d t$ does not converge weakly for $t$ fixed.

The next corollary shows that one does have weak convergence in the complement of the barrier. 
Corollary 3.3 Suppose that $\epsilon_{n}, w^{n}, u$, and $u^{n}$ are as in Proposition 3.1. For every fixed $t>0$, the sequence $\left(u^{n}(t), \partial_{t} u^{n}(t)\right)$ converges weakly to $\left(u(t), \partial_{t} u(t)\right)$ outside the barrier; that is, for every $\Phi=\left(\Phi_{1}, \Phi_{2}\right) \in H$ with supp $\Phi_{i} \cap\{L<$ $|x|<L+1\}=\varnothing, i=1,2$,

$$
\lim _{n \rightarrow \infty}\left(\left(u^{n}(t), \partial_{t} u^{n}(t)\right), \Phi\right)_{H} \longrightarrow\left(\left(u(t), \partial_{t} u(t)\right), \Phi\right)_{H} .
$$

Proof: Since the $u^{n}(t)$ and $u_{t}^{n}(t)$ are bounded in $H$, it suffices to prove the assertion for $\Phi_{i} \in C_{0}^{\infty}\left(\mathbb{R}^{3} \backslash\{L \leq|x| \leq L+1\}\right)$ since such data are dense in the desired $\Phi$. Estimate (3.1) implies that $w_{1}$ vanishes for $L \leq|x| \leq L+1$.

An integration by parts in $x$ yields

$$
\begin{aligned}
0 & =\int_{0}^{T} \int_{\mathbb{R}^{3}}\left(\partial_{t}^{2} u^{n}-\Delta_{x} u^{n}+V^{\epsilon_{n}} u^{n}\right) \Phi(x) d x \\
& =\int_{0}^{T}\left(\partial_{t}^{2} u^{n}, \Phi\right)_{L^{2}} d t+\int_{0}^{T}\left(\nabla_{x} u^{n}, \nabla_{x} \Phi\right)_{L^{2}} d t+\int_{0}^{T}\left(q(t) \chi^{\delta} u^{n}, \Phi\right)_{L^{2}} d t .
\end{aligned}
$$

The boundary terms on $|x|=L$ and $|x|=L+1$ vanish since supp $\Phi \cap\{x: L<$ $|x|<L+1\}=\varnothing$.

Proposition 3.1 implies weak convergence of $u^{n}$ in $L^{2}([0, T] ; D)$ and weak convergence of $u_{t}^{n}$ in $L^{2}\left([0, T] ; L^{2}\left(\mathbb{R}^{3}\right)\right)$ to $u$ and $u_{t}$, respectively. The fundamental theorem of calculus implies

$$
\int_{0}^{T} \partial_{t}\left(u_{t}^{n}, \Phi\right)_{L^{2}} d t=\left(u_{t}^{n}(T), \Phi\right)_{L^{2}}-\left(w_{2}^{n}, \Phi\right)_{L^{2}}
$$

Passing to the limit $n \rightarrow \infty$ yields

$$
\begin{aligned}
\lim _{n \rightarrow \infty}\left(u_{t}^{n}(T), \Phi\right)_{L^{2}}= & \left(w_{2}, \Phi\right)_{L^{2}}-\int_{0}^{T}\left(\nabla_{x} v, \nabla_{x} \Phi\right)_{L^{2}} d t \\
& -\int_{0}^{T} \int_{|x| \leq L} q(t) \chi^{\delta} v \Phi d x d t
\end{aligned}
$$

Since $\Phi$ is supported outside the barrier, a similar integration by parts yields

$$
\begin{aligned}
\left(u_{t}(T), \Phi\right)_{L^{2}}= & \left(w_{2}, \Phi\right)_{L^{2}}-\int_{0}^{T}\left(\nabla_{x} u, \nabla_{x} \Phi\right)_{L^{2}} d t \\
& -\int_{0}^{T} \int_{|x| \leq L} q(t) \chi^{\delta} u \Phi d x d t
\end{aligned}
$$

Combining (3.4) and (3.5) shows that $u_{t}^{n}(T)$ converges weakly to $u_{t}(T)$ in $L^{2}\left(\mathbb{R}^{3} \backslash\{L \leq|x| \leq L+1\}\right)$. 
Next

$$
\begin{aligned}
& \left(\nabla_{x} u^{n}(T), \nabla_{x} \Phi\right)_{L^{2}}-\left(\nabla_{x} w_{1}^{n}, \nabla \Phi\right)_{L^{2}} \\
& \quad=-\left(u^{n}(T), \Delta_{x} \Phi\right)_{L^{2}}+\left(w_{1}^{n}, \Delta_{x} \Phi\right)_{L^{2}} \\
& \quad=-\int_{0}^{T} \partial_{t}\left(u^{n}, \Delta_{x} \Phi\right)_{L^{2}} d t=-\int_{0}^{T}\left(u_{t}^{n}, \Delta_{x} \Phi\right)_{L^{2}} d t
\end{aligned}
$$

The same computation with $u^{n}$ replaced by $u$ yields

$$
\left(\nabla_{x} u(T), \nabla_{x} \Phi\right)_{L^{2}}=\left(\nabla_{x} w_{1}, \nabla \Phi\right)_{L^{2}}-\int_{0}^{T}\left(u_{t}, \Delta_{x} \Phi\right)_{L^{2}} d t
$$

In (3.6) use the weak convergence of $u_{t}^{n}$ in $L^{2}\left([0, T] ; L^{2}\left(\mathbb{R}^{3}\right)\right)$ and that of $w_{1}^{n}$ in $D$ to find

$$
\lim _{n \rightarrow \infty}\left(\nabla_{x} u^{n}(T), \nabla_{x} \Phi\right)_{L^{2}}=\left(\nabla_{x} w_{1}, \nabla_{x} \Phi\right)_{L^{2}}-\int_{0}^{T}\left(u_{t}, \Delta_{x} \Phi\right)_{L^{2}} d t
$$

Comparing with (3.7) completes the proof of weak convergence.

\section{Weak Resolvent Convergence}

Denote by $S^{\epsilon}(t)=U^{\epsilon}(t, 0)$ the map from Cauchy data at time 0 to Cauchy data at time $t$ for the solutions of the wave equation with periodic potential $V^{\epsilon}$. Next denote by $S_{0}^{\epsilon}(t)$ the map from Cauchy data at time 0 to Cauchy data at time $t$ for the wave equation with time-independent potential $b^{\epsilon}(x)$. Finally, given $w=$ $\left(w_{1}, w_{2}\right) \in H$ such that both components vanish on $\{x: L \leq|x| \leq L+1\}$, introduce the operator

$$
D(t) w:=\left(u(t), u_{t}(t)\right)
$$

where $u$ is as in Proposition 3.1. More precisely, $D(t) w=0$ for $L \leq|x| \leq L+1$, and if $D(t) w=\left(u(t), \partial_{t} u(t)\right)$, then $u$ is the solution of the Dirichlet problems in $|x| \leq L$ and $|x| \geq L+1$, respectively, for $\partial_{t}^{2}-\Delta_{x}+q(t) \chi^{\delta}(x)$ and $\partial_{t}^{2}-\Delta_{x}$. Notice that for $w$ with the properties above, if $z \notin \operatorname{spec} D(t)$, we have $(D(t)-z I)^{-1} w=$ $\left(g_{1}, g_{2}\right)$, where $g_{1}$ and $g_{2}$ vanish for $L \leq|x| \leq L+1$.

The operator $S_{0}^{\epsilon}(t)$ is not defined in terms of $U_{0}(t)$. Since $b^{\epsilon}(x)$ is independent of $t$, the operator $S_{0}^{\epsilon}(t)$ is unitary in the $\epsilon$-dependent norm $\|\cdot\|_{\epsilon}$. More precisely, for a bounded operator $A \in \mathcal{L}(H)$ we use the operator norm

$$
\|A\|_{\epsilon}=\sup _{f \neq 0} \frac{\|A f\|_{\epsilon}}{\|f\|_{\epsilon}}
$$

$\|\cdot\|_{\epsilon}$ being the norm in $H$ related to $b^{\epsilon}(x)$ defined in Section 3.

Therefore, for $|z|>1$ we have

$$
\left\|\left(S_{0}^{\epsilon}(t)-z I\right)^{-1}\right\|_{\epsilon} \leq \frac{1}{|z|-1} .
$$


The time-dependent lower-order term $q(t) \chi^{\delta}(x)$ is a bounded perturbation. Therefore,

$$
\forall \underline{T}, \quad \exists C, \quad \forall t \in[0, \underline{T}], \quad \epsilon \in] 0,1], \quad \varphi \in H, \quad\left\|S^{\epsilon}(t) \varphi\right\|_{\epsilon} \leq C\|\varphi\|_{\epsilon},
$$

with $C>0$ depending on $\underline{T}>0$ but not on $\epsilon$.

Lemma 4.1 For each $\epsilon>0, \underline{T}>0$, the operator $S^{\epsilon}(\underline{T})-S_{0}^{\epsilon}(\underline{T}) \in \mathcal{L}(H)$ is compact. In addition, if $w^{n} \rightarrow 0$ weakly in $H$ and satisfies (3.1), then for $n \rightarrow \infty$,

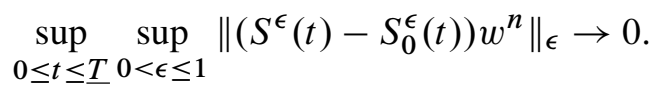

Proof: Fix $0<\epsilon \leq 1$ and consider the solutions $u_{n}$ and $v_{n}$ of the Cauchy problems

$$
\begin{aligned}
\left(\partial_{t}^{2}-\Delta_{x}\right) u_{n}+V^{\epsilon} u_{n} & =0, & \left(u_{n}, \partial_{t} u_{n}\right)(0, x) & =w^{n}, \\
\left(\partial_{t}^{2}-\Delta_{x}\right) v_{n}+b^{\epsilon} v_{n} & =0, & \left(v_{n}, \partial_{t} v_{n}\right)(0, x) & =w^{n} .
\end{aligned}
$$

Then $\left(u_{n}-v_{n}\right)$ satisfies

$$
\left(\partial_{t}^{2}-\Delta+b^{\epsilon}\right)\left(u_{n}-v_{n}\right)=-q(t) \chi^{\delta}(x) u_{n}, \quad\left(u_{n}-v_{n}\right)(0)=\partial_{t}\left(u_{n}-v_{n}\right)(0)=0 .
$$

The energy identity implies that for $0 \leq \tau \leq \underline{T}$,

$$
\left\|u_{n}(\tau, x)-v_{n}(\tau, x)\right\|_{\epsilon}^{2}=-2 \operatorname{Re} \int_{0}^{\tau} \int_{\mathbb{R}^{3}} u_{n} q \chi^{\delta} \overline{\partial_{t}\left(u_{n}-v_{n}\right)} d t d x .
$$

Proposition 3.1 implies that

$$
u_{n} \rightarrow 0 \quad \text { weak-star in } L^{\infty}([0, \underline{T}] ; D),
$$

and

$$
\partial_{t}\left(u_{n}-v_{n}\right) \rightarrow 0 \quad \text { weak-star in } L^{\infty}\left([0, \underline{T}] ; L^{2}\left(\mathbb{R}^{3}\right)\right) .
$$

Since $q \chi^{\delta}$ has compact spatial support, it follows that

$$
q \chi^{\delta} u_{n} \rightarrow 0 \quad \text { strongly in } L^{2}\left([0, T] ; L^{2}\left(\mathbb{R}^{3}\right)\right) .
$$

On the other hand,

$$
\partial_{t}\left(u_{n}-v_{n}\right) \rightarrow 0 \quad \text { weakly in } L^{2}\left([0, T] ; L^{2}\left(\mathbb{R}^{3}\right)\right),
$$

and we deduce

$$
\int_{\mathbb{R}^{3}} u_{n} q \chi^{\delta} \overline{\partial_{t}\left(u_{n}-v_{n}\right)} d x \rightarrow 0 \quad \text { in } L^{1}([0, \underline{T}] ; \mathbb{C}) .
$$

This together with (4.1) completes the proof. 
Proposition 4.2 Let $K \subset\{z \in \mathbb{C}:|z|>1\}$ be a compact set disjoint from

$$
\operatorname{spec} D(T) \cup\left(\bigcup_{0<\epsilon \leq \epsilon_{1}} \operatorname{spec} S^{\epsilon}(T)\right) .
$$

Then there exist $\epsilon_{0}>0$ and $C_{0}>0$ so that

$$
\left.\forall \epsilon \in] 0, \epsilon_{0}\right], \quad z \in K, \quad\left\|\left(S^{\epsilon}(T)-z I\right)^{-1}\right\|_{\epsilon} \leq C_{0} .
$$

PROOF: In $\{z \in \mathbb{C}:|z| \gg 1\}$, the operators $S^{\epsilon}(T)-z I$ are compact perturbations of the invertible operators $S_{0}^{\epsilon}(T)-z I$, both depending analytically on $z$. The analytic Fredholm theory implies that for each $\epsilon,\left(S^{\epsilon}(T)-z I\right)^{-1}$ has a meromorphic continuation in $\{z \in \mathbb{C}:|z|>1\}$.

If the proposition were false, there would exist $\epsilon_{n} \rightarrow 0, z_{n} \in K$, and $w_{n}$ with

$$
\left\|w_{n}\right\|_{\epsilon_{n}}=1, \quad\left\|\left(S^{\epsilon_{n}}(T)-z_{n} I\right) w_{n}\right\|_{\epsilon_{n}} \rightarrow 0 .
$$

Both parts of (4.3) give strong control in the barrier. Passing to a subsequence, we may suppose that $w_{n}$ converges weakly to a limit $w$ in $H$ and $z_{n} \rightarrow z \in K$. As in Proposition 3.1, we deduce that $w=0$ for $L \leq|x| \leq L+1$, and the definition of $D(T)$ given in the beginning of this section implies $(D(T)-z I) w=0$ for $L \leq|x| \leq L+1$.

We claim that $(D(T)-z I) w=0$ for every $x$. Corollary 3.3 implies that $S^{\epsilon_{n}}(T) w_{n}$ converges weakly to $D(T) w$ outside of the barrier, and the second expression in (4.3) yields

$$
\left\|S^{\epsilon_{n}}(T) w_{n}-z_{n} w_{n}\right\|_{\epsilon_{n}} \rightarrow 0
$$

Passing to the limit $n \rightarrow \infty$ shows that $D(T) w-z w=0$ for $x \in \mathbb{R}^{3} \backslash\{L \leq|x| \leq$ $L+1\}$, and the claim is established.

Consequently, $D(T) w=z w$ for all $x \in \mathbb{R}^{3}$. Since $z$ is not in the spectrum of $D(T)$, it follows that $w=0$. Next

$$
\begin{aligned}
\left\|\left(S^{\epsilon_{n}}(T)-z I\right) w_{n}\right\|_{\epsilon_{n}} & =\left\|\left(S_{0}^{\epsilon_{n}}(T)-z I\right) w_{n}+\left(S^{\epsilon_{n}}(T)-S_{0}^{\epsilon_{n}}(T)\right) w_{n}\right\|_{\epsilon_{n}} \\
& \geq\left\|\left(S_{0}^{\epsilon_{n}}(T)-z I\right) w_{n}\right\|_{\epsilon_{n}}+\left\|\left(S^{\epsilon_{n}}(T)-S_{0}^{\epsilon_{n}}(T)\right) w_{n}\right\|_{\epsilon_{n}} .
\end{aligned}
$$

Lemma 4.1 implies that the second summand tends to 0 , so

$$
\left\|\left(S^{\epsilon_{n}}(T)-z I\right) w_{n}\right\|_{\epsilon_{n}} \geq(|z|-1)\left\|w_{n}\right\|_{\epsilon_{n}}+o(1)=(|z|-1)+o(1) .
$$

This contradicts (4.3) and therefore proves the proposition.

\section{Remark 4.3.}

(i) It follows that for all $\varphi$ and $0<\epsilon \leq \epsilon_{0}$,

$$
\left\|\left(S^{\epsilon}(T)-z I\right)^{-1} \varphi\right\|_{H} \leq\left\|\left(S^{\epsilon}(T)-z I\right)^{-1} \varphi\right\|_{\epsilon} \leq C_{0}\|\varphi\|_{\epsilon} \leq \frac{C}{\sqrt{\epsilon}}\|\varphi\|_{H} .
$$

Therefore

$$
\left\|\left(S^{\epsilon}(T)-z I\right)^{-1}\right\|_{\mathcal{L}(H)} \leq \frac{C}{\sqrt{\epsilon}}
$$


On the other hand, for $\varphi$ vanishing in $L \leq|x| \leq L+1$, we get

$$
\left\|\left(S^{\epsilon}(T)-z I\right)^{-1} \varphi\right\|_{H} \leq C_{0}\|\varphi\|_{H} .
$$

(ii) The compactness of the lower-order term is related to the fact that it does not affect the leading-order terms in the propagation of singularities. On the negative side, this eliminates one possible strategy for amplification, and it prevents us from using microlocal techniques. It is a key ingredient in the proofs of this section.

Proposition 4.4 Suppose that both components of $\varphi \in H$ vanish for $L \leq|x| \leq$ $L+1$, and $z \in\{z \in \mathbb{C}:|z|>1\}$ satisfies

$$
z \notin \operatorname{spec} D(T) \cup\left(\bigcup_{0<\epsilon \leq \epsilon_{0}} \operatorname{spec} S^{\epsilon}(T)\right) .
$$

Then

$$
\lim _{\epsilon \rightarrow 0}\left(S^{\epsilon}(T)-z I\right)^{-1} \varphi=(D(T)-z I)^{-1} \varphi \quad \text { weakly in } H .
$$

ProOF: Let

$$
\psi^{\epsilon}:=\left(S^{\epsilon}(T)-z I\right)^{-1} \varphi .
$$

Since $\|\varphi\|_{\epsilon}$ is bounded independently of $\epsilon$, the resolvent estimate (4.2) implies that uniformly in $\epsilon$ we have

$$
\left\|\psi^{\epsilon}\right\|_{\epsilon}=\left\|\left(S^{\epsilon}(T)-z I\right)^{-1} \varphi\right\|_{\epsilon} \leq C .
$$

Since $\left\|\psi^{\epsilon}\right\|_{H} \leq\left\|\psi^{\epsilon}\right\|_{\epsilon}$, every subsequence has a subsequence that is weakly convergent. Denote by $\psi$ the weak limit in $H$ of such a weakly convergent subsequence. Clearly, $\psi$ vanishes for $L \leq|x| \leq L+1$. It suffices to show that $\psi=(D(T)-z I)^{-1} \varphi$.

Applying $S^{\epsilon}(T)-z I$ to (4.5), we find

$$
\left(S^{\epsilon}(T)-z I\right) \psi^{\epsilon}=\varphi .
$$

Since $\psi$ vanishes for $L \leq|x| \leq L+1$, an application of Corollary 3.3 shows that $S^{\epsilon}(T) \psi^{\epsilon}$ converges weekly to $D(T) \psi$ in $D_{0} \times L^{2}\left(\mathbb{R}^{3} \backslash\{L \leq|x| \leq L+1\}\right)$. Thus passing to the limit $\epsilon \rightarrow 0$, we deduce

$$
(D(T)-z I) \psi=\varphi .
$$

Therefore $\psi=(D(T)-z I)^{-1} \varphi$ and the proof is complete.

\section{Main Theorem}

Our construction in Section 2 shows that for $r>0$ small enough the operator $K^{\delta}(T)$ with fixed $\delta>0$ has exactly one eigenvalue $z_{1}$ inside the disc $D_{r}=\{z \in$ $\left.\mathbb{C}:\left|z-z_{1}\right| \leq r\right\}$ with eigenfunction $\varphi$. The next result is more precise than Theorem 1.1 and Corollary 1.2. 
TheOREM 5.1 Suppose that $\gamma=\left\{z \in \mathbb{C}:\left|z-z_{1}\right|=r,|z|>1\right\}$ is a circle disjoint from the spectrum of $K^{\delta}(T)$ so that $K^{\delta}(T)$ has exactly one eigenvalue $z_{1}$ in $D_{r}$. Then there exists an $\epsilon_{0}$ so that for $0<\epsilon \leq \epsilon_{0}$ the operator $S^{\epsilon}(T)$ has an eigenvalue in $D_{r}$. For the same values of $\epsilon$, problem (1.1) with potential $V^{\epsilon}(t, x)$ has a resonance in $D_{r}$.

Proof: If $K^{\delta}(T) \varphi=z_{1} \varphi, \varphi \neq 0$, it is clear that we can extend $\varphi$ as 0 outside $B_{L}$ and $D(T) \varphi=z_{1} \varphi$. First we prove that $S^{\epsilon}(T)$ has an eigenvalue. If $S^{\epsilon}(T)$ has an eigenvalue on $\gamma$, the assertion holds, so we suppose that $S^{\epsilon}(T)$ has no eigenvalues on $\gamma$.

For $|z| \gg 1$ we write

$$
(D(T)-z I)^{-1} \varphi=f_{1}(z)+f_{2}(z),
$$

where $f_{1}(z) \in H$ vanishes outside $B_{L}$, while $f_{2}(z) \in H$ vanishes for $|x| \leq L+1$. Applying $D(T)-z I$, we deduce $(D(T)-z I) f_{2}(z)=0$, and since the propagator of the Dirichlet problem in $\left\{x \in \mathbb{R}^{3}:|x| \geq L+1\right\}$ has no eigenvalues $z,|z|>1$, we obtain $f_{2}(z)=0$. Consequently,

$$
(D(T)-z I)^{-1} \varphi=\left(K^{\delta}(T)-z I\right)^{-1} \varphi=f_{1}(z) .
$$

For $z \in \gamma$, by analytic continuation we deduce

$$
(D(T)-z I)^{-1} \varphi=\left(K^{\delta}(T)-z I\right)^{-1} \varphi
$$

hence the operator $D(T)$ has no eigenvalues $z \in \gamma$. Next

$$
\frac{1}{2 \pi i} \oint_{\gamma}(D(T)-z I)^{-1} \varphi d z=\frac{1}{2 \pi i} \oint_{\gamma}\left(K^{\delta}(T)-z I\right)^{-1} \varphi d z=\varphi .
$$

Since both components of $\varphi$ vanish on $\{L \leq|x| \leq L+1\}$, we have weak resolvent convergence from Proposition 4.4. Moreover,

$$
\oint_{\gamma}\left(\varphi,(D(T)-z I)^{-1} \varphi\right)_{H} d z \neq 0
$$

Estimate (4.2) implies that there is a constant $C$ so that

$$
\sup _{0<\epsilon \leq \epsilon_{0}} \sup _{z \in \gamma}\left|\left(\varphi,\left(S^{\epsilon}(T)-z I\right)^{-1} \varphi\right)_{H}\right| \leq C .
$$

Together with the weak convergence from Proposition 4.4, the dominated convergence theorem implies that

$$
\lim _{\epsilon \rightarrow 0} \oint_{\gamma}\left(\varphi,\left(S^{\epsilon}(T)-z I\right)^{-1} \varphi\right)_{H} d z=\oint_{\gamma}\left(\varphi,(D(T)-z I)^{-1} \varphi\right)_{H} d z \neq 0 .
$$

Therefore, there exists an $\epsilon_{1}>0$ so that for $0<\epsilon \leq \epsilon_{1}$,

$$
\oint_{\gamma}\left(\varphi,\left(S^{\epsilon}(T)-z I\right)^{-1} \varphi\right)_{H} d z \neq 0 \text {. }
$$


Consequently, for these $\epsilon$, the operator $\left(S^{\epsilon}(T)-z I\right)^{-1}$ is not analytic in $D_{r}$. Hence $S^{\epsilon}(T)$ has an eigenvalue inside $D_{r}$.

Theorem 5.4.1 of [12] shows that every eigenvalue $z_{\epsilon}$ of $U^{\epsilon}(T, 0)$ with $\left|z_{\epsilon}\right|>1$ is automatically a resonance for the problem (1.1) with $V^{\epsilon}(t, x)$. Moreover, the algebraic multiplicities of the eigenvalue and the resonance are the same.

We give an independent proof of the weaker fact that there is a resonance in $D_{r}$ based on the second definition of resonances as the poles of the meromorphic continuation of the cutoff resolvent given in Section 1 .

Fix $\epsilon$ so that $0<\epsilon \leq \epsilon_{1}$ and (5.1) holds. Let $\Phi \in C_{0}^{\infty}\left(\mathbb{R}^{3}\right)$ be a cutoff function such that $\Phi(x)=1$ for $x \in B_{L}$. Then

$$
\oint_{\gamma}\left(\Phi \varphi,\left(S^{\epsilon}(T)-z I\right)^{-1} \Phi \varphi\right)_{H} d z=\oint_{\gamma}\left(\varphi, \Phi\left(S^{\epsilon}(T)-z I\right)^{-1} \Phi \varphi\right)_{H} d z \neq 0 .
$$

Therefore the cutoff resolvent $\Phi\left(S^{\epsilon}(T)-z I\right)^{-1} \Phi$ is not analytic in $D_{r}$, which proves the existence of a resonance in $D_{r}$.

Acknowledgment. The research of Jeffrey Rauch was partially supported by the National Science Foundation under NSF Grant DMS-0405899.

\section{Bibliography}

[1] Bachelot, A.; Petkov, V. Existence des opérateurs d'ondes pour les systèmes hyperboliques avec un potentiel périodique en temps. Ann. Inst. H. Poincaré Phys. Théor. 47 (1987), no. 4, 383-428.

[2] Beale, J. T. Scattering frequencies of resonators. Comm. Pure Appl. Math. 26 (1973), 549-563.

[3] Bony, J.-F.; Petkov, V. Resonances for non-trapping time-periodic perturbations. J. Phys. A 37 (2004), no. 40, 9439-9449.

[4] Colombini, F.; Rauch, J. Smooth localized parametric resonance for wave equations. J. Reine Angew. Math., forthcoming.

[5] Cooper, J. Asymptotic behavior for the vibrating string with a moving boundary. J. Math. Anal. Appl. 174 (1993), no. 1, 67-87.

[6] Cooper, J. Parametric resonance in wave equations with a time-periodic potential. SIAM J. Math. Anal. 31 (2000), no. 4, 821-835 (electronic).

[7] Cooper, J.; Menzala, G. P.; Strauss, W. On the scattering frequencies of time-dependent potentials. Math. Methods Appl. Sci. 8 (1986), no. 4, 576-584.

[8] Cooper, J.; Strauss, W. Energy boundedness and decay of waves reflecting off a moving obstacle. Indiana Univ. Math. J. 25 (1976), no. 7, 671-690.

[9] Cooper, J.; Strauss, W. Scattering of waves by periodically moving bodies. J. Funct. Anal. 47 (1982), no. 2, 180-229.

[10] Lax, P. D.; Phillips, R. S. Scattering theory. 2nd ed. Pure and Applied Mathematics, 26. Academic, Boston, 1989.

[11] Magnus, W.; Winkler, S. Hill's equation. Interscience Tracts in Pure and Applied Mathematics, 20. Interscience Wiley, New York-London-Sydney, 1966.

[12] Petkov, V. Scattering theory for hyperbolic operators. Studies in Mathematics and Its Applications, 21. North-Holland, Amsterdam, 1989. 
[13] Petkov, V. Local energy decay and Strichartz estimates for the wave equation with time-periodic perturbations. Phase space analysis of partial differential equations, 267-285. Progress in Nonlinear Differential Equations and Their Applications, 69. Birkhäuser, Boston, 2006.

[14] Popov, G.; Rangelov, T. Exponential growth of the local energy for moving obstacles. Osaka J. Math. 26 (1989), no. 4, 881-895.

[15] Strauss, W. A.; Majda, G.; Wei, M. S. Imaginary poles of radial potentials. Mat. Apl. Comput. 6 (1987), no. 1, 17-23.

[16] Vaŭnberg, B. R. On the local energy of solutions of exterior mixed problems that are periodic with respect to $t$. Trudy Moskov. Mat. Obshch. 54 (1992), 213-242, 279; translation in Trans. Moscow Math. Soc. (1993), 191-216.

[17] Wei, M. S.; Majda, G. Numerical computation of the scattering frequencies for a cylindrically symmetric potential. SIAM J. Sci. Comput. 14 (1993), no. 2, 295-309.

[18] Wei, M. S.; Majda, G. Relationships between a potential and its scattering frequencies. SIAM J. Appl. Math. 55 (1995), no. 4, 1094-1116.

\section{FERRUCCIO COLOMBini}

Università di Pisa

Dipartimento di Matematica

Largo Bruno Pontecorvo 5

56127 Pisa

ITALY

E-mail: colombinie

$$
\text { dm.unipi.it }
$$

JEFFREY RAUCH

University of Michigan

Department of Mathematics

2074 East Hall

530 Church Street

Ann Arbor, MI 48109-1043

E-mail: rauch@umich.edu

Received December 2007.

\author{
Vesselin Petkov \\ Université Bordeaux 1 \\ Institut de Mathématiques \\ Bât A33 \\ 351 cours de la Libération \\ F-33405 Talence \\ FRANCE
}

E-mail: petkov@math.

$$
\text { u-bordeaux1.fr }
$$

